\title{
Characterization of Interfacial Bonding Mechanism for Graphene- Modified Powder Metallurgy Nickle-Based Superalloy
}

\author{
Jin-Wen Zou ${ }^{1,2} \cdot$ Xiao-Feng Wang ${ }^{1,2} \cdot$ Jie Yang ${ }^{1,2} \cdot$ Chuan-Bo $\mathrm{Ji}^{1,2} \cdot$ Xu-Qing Wang ${ }^{1,2} \cdot$ Xian-Qiang Fan $^{3} \cdot$ \\ Zhi-Peng Guo ${ }^{3}$
}

Received: 21 September 2017/Revised: 22 October 2017/Published online: 29 January 2018

(C) The Chinese Society for Metals and Springer-Verlag GmbH Germany, part of Springer Nature 2018

\begin{abstract}
A modified FGH96 superalloy using $0.1 \mathrm{wt} \%$ graphene was successfully prepared using the wet mixing method. The interfacial bonding mechanism between the graphene and the superalloy matrix was characterized using optical microscope, scanning electronic microscope, transmission electronic microscope and X-ray tomography. The results revealed that the graphene could be dispersed uniformly inside the matrix of the superalloy, and the bonding interface between graphene and the superalloy showed a rather diffusion instead of abrupt distinction, suggesting that the interface was formed via chemical fusion rather than a mechanical combination. The uniform dispersity of the graphene inside the superalloy matrix could improve the tensile properties significantly, including tensile strength, plasticity and yield strength. The existence of the graphene at the fracture surface further verified that the graphene could increase the effective bearing force of the material during the tensile test.
\end{abstract}

Keywords Powder metallurgical (PM) superalloy · Graphene - Interfacial binding mechanism - Mechanical properties · Characterization

\section{Introduction}

Graphene, a single-layer carbon atom material found by Novoselov et al. [1] with thickness of $0.3354 \mathrm{~nm}$, is the thinnest material at present known to human beings. Graphene is an allotrope of carbon in the two-dimensional, atomic-scale, hexagonal lattice form in which one atom forms each vertex [2-4]. Owning to its special structure, graphene possesses excellent mechanical, electrical and thermal properties; particularly, $130 \mathrm{GPa}$ tensile strength

Available online at http://link.springer.com/journal/40195

Xiao-Feng Wang

wangxiaofeng_0404@163.com

1 AECC Beijing Institute of Aeronautical Materials, Beijing 100089, China

2 Science and Technology on Advanced High Temperature Structural Materials Laboratory, Beijing Institute of Aeronautical Materials, Beijing 100089, China

3 School of Materials Science and Engineering, Tsinghua University, Beijing 100089, China and $1.02 \mathrm{TPa}$ elastic modulus make graphene an ideal reinforcement for composite materials [5-7].

As a second phase, addition of the graphene could increase significantly the mechanical properties of the metallic alloys. Extensive studies have been performed on improving the mechanical properties of the materials by adding the graphene, but most attention so far was focused on polymers and nonmetallic alloys because of its low density, aggregation and interface reaction between graphene and matrix [8-15]. During last 10 years, carbon nanotubes (CNTs) have been widely added into metal matrix to enhance the mechanical properties of the pure metal. However, uniform dispersion of the CNTs in the metal matrix was proved to be difficult and challenging $[16,17]$. Because graphene has several advantages over CNTs due to its two-dimensional structure, studies have been performed to modify metal composite using graphene [18-25]. Recent studies showed that by ball milling and powder metallurgy, an addition of $0.3 \mathrm{wt} \%$ graphene uniformly dispersed in the aluminum alloy could increase the tensile strength and yield strength up to 25 and $58 \%$, respectively [26]. A graphene-modified magnesium-based composite material was manufactured by conducting 
ultrasound processing in liquid and mechanical stirring in solid, and it was shown that the graphene was dispersed uniformly inside the composite matrix and a significant increase of the mechanical properties was achieved [27]. And also, aluminum composites reinforced with graphene nanosheets (GNSs) were fabricated through a feasible methodology based on flake powder metallurgy. The tensile strength of $249 \mathrm{MPa}$ was achieved in the $\mathrm{Al}$ composite reinforced with only $0.3 \mathrm{wt} \%$ GNSs, which is $62 \%$ enhancement over the unreinforced Al matrix [28].

Because of the significant difference of the density, limited dispersity and chemical reaction at the interface during thermal processing, the mixture of graphene and the superalloy matrix was shown difficult. On the other hand, if in some way we can implant these advantage features of graphene, e.g., high strength and high specific modulus with the current superalloy, it is highly likely that the mechanical properties of the final composite material could be enhanced significantly.

In this work, we employed the so-called wet mixing method to disperse the graphene inside the nickel-based FGH96 superalloy. The material was then prepared by hot isostatic pressing (HIP), hot extrusion (HEX), hot isothermal forging (HIF) and heat treatment (HT). To disclose the underlying bonding mechanism between the graphene and the superalloy matrix, especially at the phase interface, a systematical characterization using optical microscope (OM), scanning electronic microscope (SEM), transmission electronic microscope (TEM) and X-ray imaging technique was performed, and the improvement of the mechanical properties was discussed in detail.

\section{Experimental}

The FGH96 superalloy powder was prepared via argon atomization after melting the ingot using vacuum induction heating. The chemical composition of this alloy is shown in Table 1. The graphene, in a form of oxide nanolayers, was then dispersed uniformly inside the superalloy powders using the wet mixing method. Vacuum processing was then performed to the modified FGH96 superalloy to remove the absorbed gas at the interface and transform the oxide graphene layer into graphene.

The modified FGH96 superalloy powder was then sealed inside a stainless steel container, which was then processed with HIP, HEX, hot isothermal forging (HIF) and HT including solution and aging. Experimental characterization using OM, SEM, TEM and X-ray imaging technique was then performed on the microstructure of the modified superalloy. The TEM samples were thinned firstly by mechanically grinding to less than $40 \mu \mathrm{m}$ followed by ion beam thinning with Gatan 691 for $4 \mathrm{~h}$. All mechanical tests were performed on a universal tensile testing machine.

Cylindrical samples with $2 \mathrm{~mm}$ in diameter and $1 \mathrm{~cm}$ in length were scanned using a X-ray tomography machine namely Nanotom $m$ (manufactured by GE). During the $\mathrm{X}$-ray tomography experiment, a voltage of $120 \mathrm{kV}$ and current of $80 \mu \mathrm{A}$ were employed to achieve a better contrast between the different phases. For each scan, the sample was rotated with $360^{\circ}$ and a total of 1000 projections of image were collected, together with dark field (without X-ray penetration) and flat field images (without samples) for further image processing. The spatial resolution of the images was $0.833 \mu \mathrm{m}$. The $3 \mathrm{D}$ reconstruction and rendering of the images were then performed using a software namely volume graphic. Image processing techniques such as contrast conditioning, thresholding and smoothing were applied during the data processing, details of which can be obtained from [29].

\section{Results and Discussion}

\subsection{Existence of the Graphene}

Figure 1 shows the graphene morphologies and diffraction patterns extracted using high-resolution TEM (HRTEM). As shown in Fig. 1a, b, the graphene existed in a twodimensional, atomic-scale, hexagonal lattice form in which one atom forms each vertex. This hexagonal structure, or "honeycomb" morphology, can also be observed clearly in the zoom-in area of the graphene surface, as shown in Fig. 1c. The transverse section of the graphene as shown in Fig. 1d exhibited a multilayer structure. It is worth stressing that the existence of a multilayer graphene could easily bring out clustering, i.e., blocking further mixing with the alloy matrix.

Figure $2 \mathrm{a}, \mathrm{b}$ shows the OM images of the surface morphologies of the modified FGH96 superalloy powder with graphene. After HIP, the graphene existed in a transparent, wrinkle, feather-like morphology in-between the superalloy particles. It could be also seen that part of the graphene was absorbed and attached to the powder surface.

Comparing with HIP, conducting the thermal processing including HEX, HIF and HT did not change the existing
Table 1 Chemical composition of FGH96 (wt\%)

\begin{tabular}{lllllllllll}
\hline $\mathrm{C}$ & $\mathrm{Cr}$ & $\mathrm{Co}$ & $\mathrm{Mo}$ & $\mathrm{W}$ & $\mathrm{Al}$ & $\mathrm{Ti}$ & $\mathrm{Nb}$ & $\mathrm{B}$ & $\mathrm{Zr}$ & $\mathrm{Ni}$ \\
\hline 0.030 & 16.000 & 13.000 & 4.000 & 4.000 & 2.200 & 3.700 & 0.800 & 0.011 & 0.036 & Bal. \\
\hline
\end{tabular}



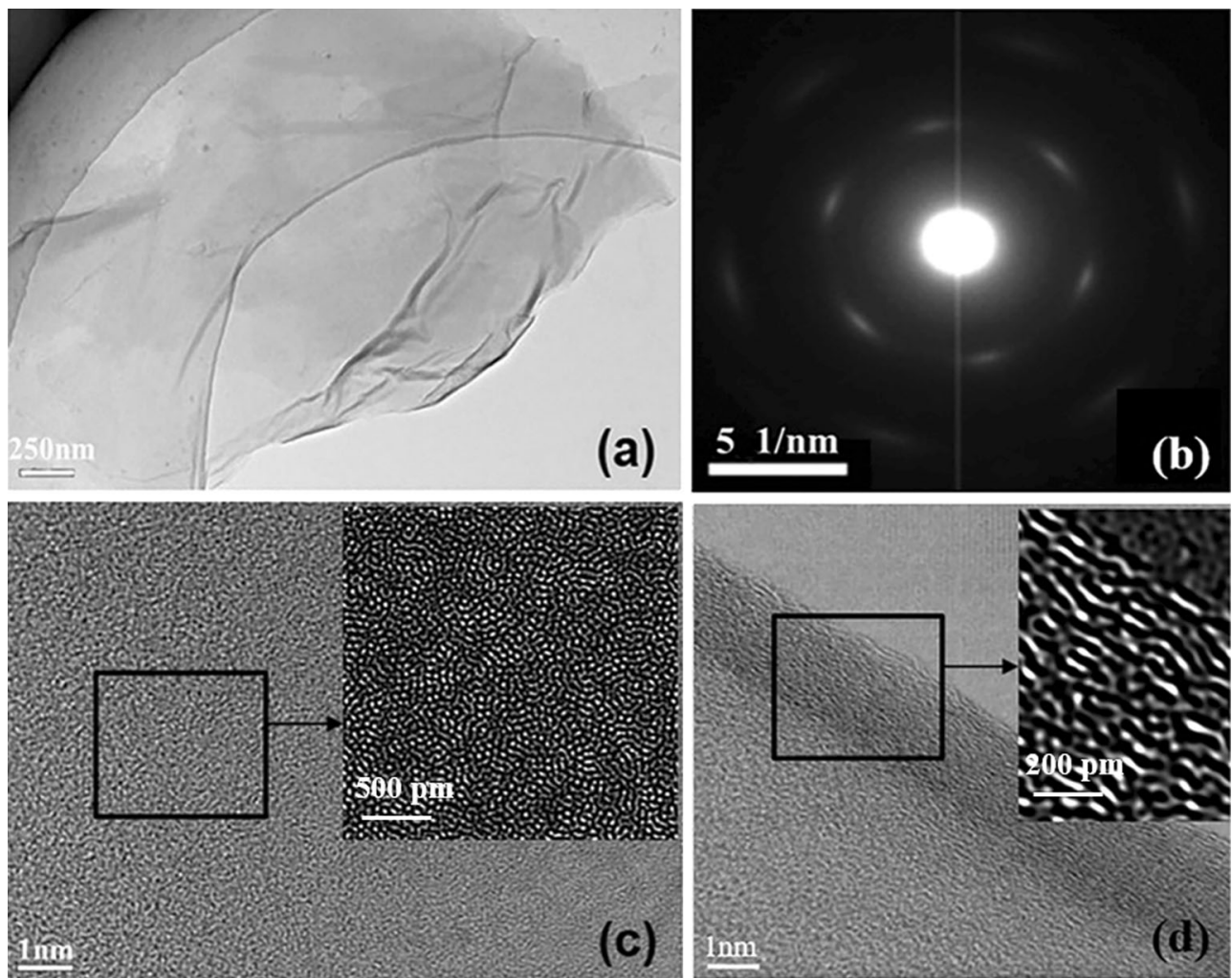

Fig. 1 HRTEM images of graphene morphology $\mathbf{a}$ and diffraction pattern $\mathbf{b}$, local zoom-in area of graphene surface $\mathbf{c}$ and transverse section of graphene layer $\mathbf{d}$
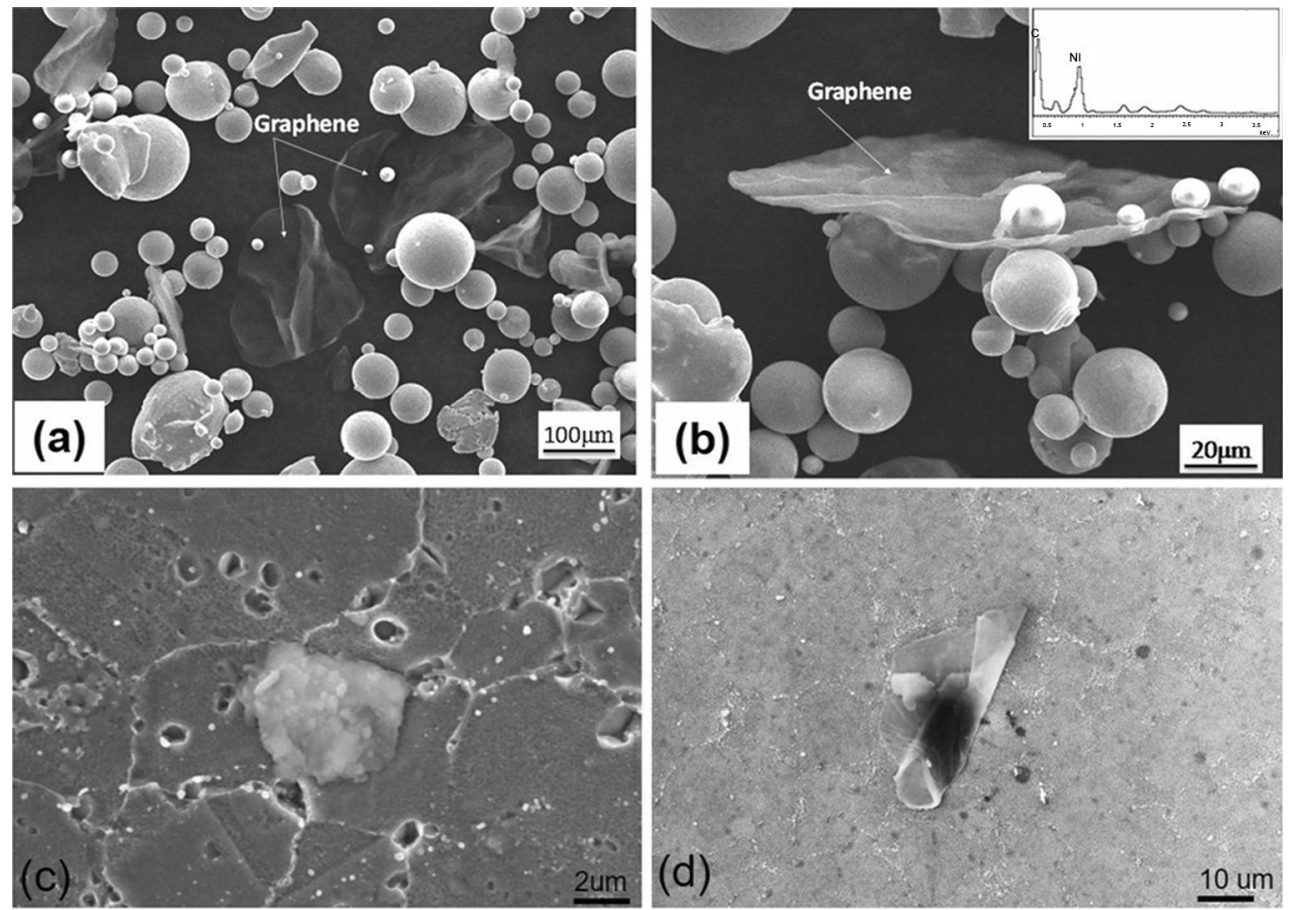

Fig. 2 Low- a and high-magnification b surface morphologies of modified FGH96 powder with graphene, low $\mathbf{c}$ and high-magnification d morphology and distribution of graphene after HIP, HEX, HIF and HT 


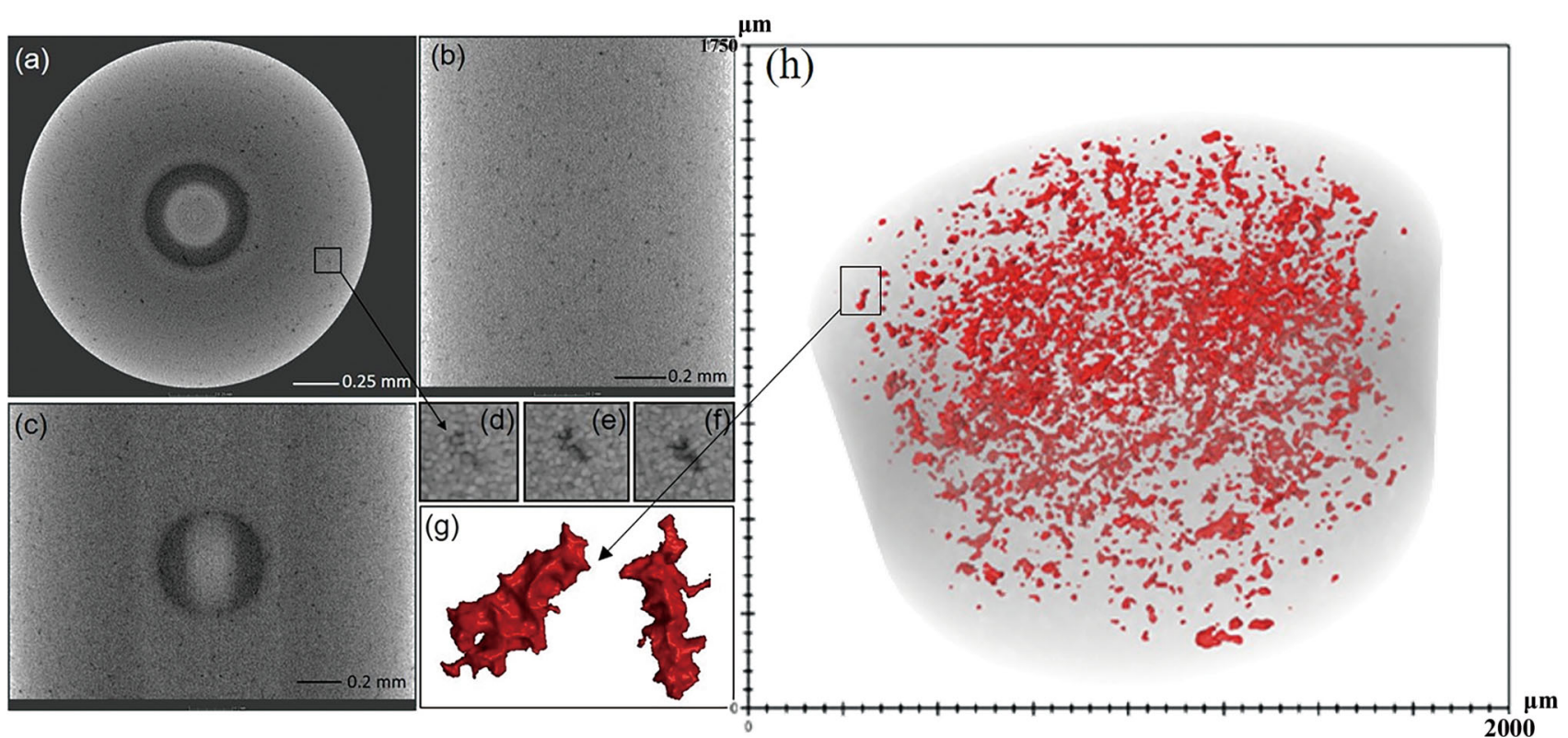

Fig. 3 Characterization of distribution of graphene inside superalloy matrix using X-ray tomography: a-c internal structures in three perpendicular view directions; d-f morphologies of typical graphene within three different image slices; $\mathbf{g}$ reconstructed 3D morphology of grapheme; $\mathbf{h}$ 3D distribution of graphene inside cylindrical sample
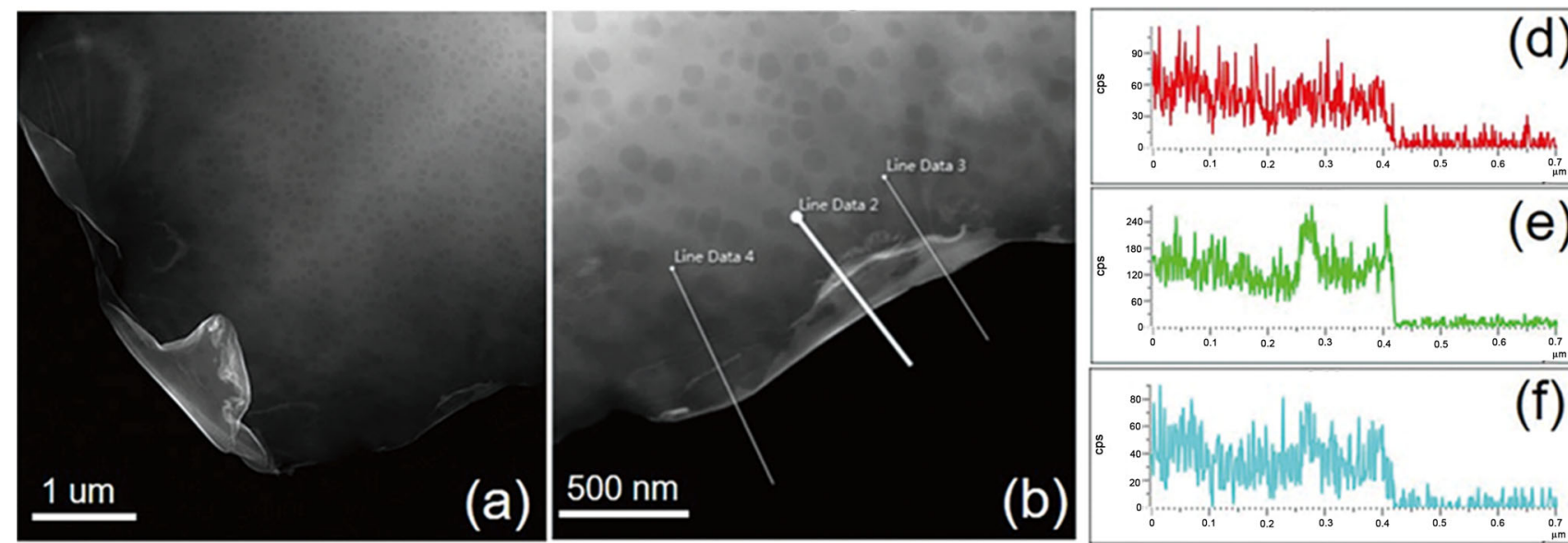

(d)
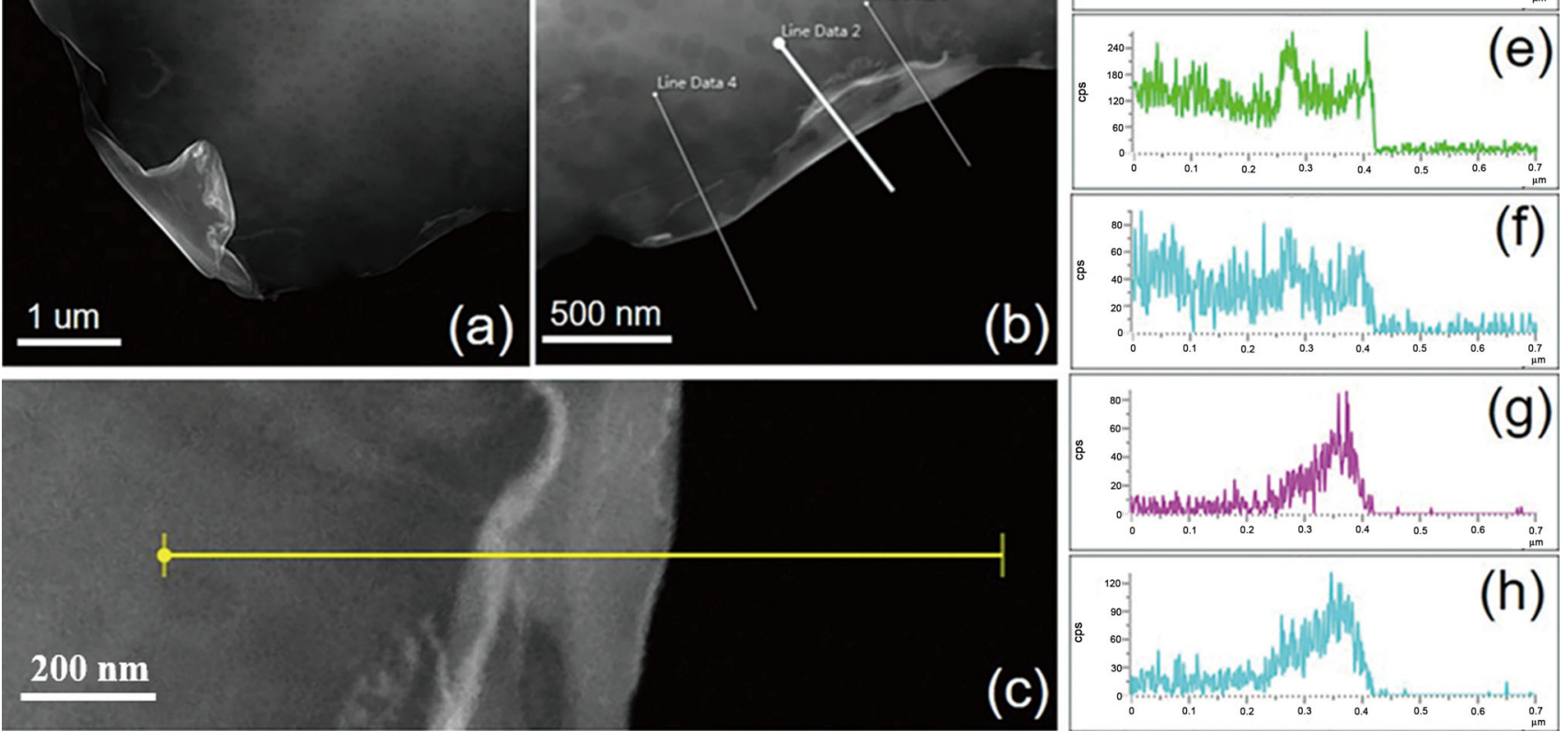

Fig. 4 HRTEM image of graphene at one edge of the sample: $\mathbf{a}, \mathbf{b}$ graphene morphologies at two different viewpoints; chemical composition of matrix elements was measured using EDS along the line as indicated in $\mathbf{c}$ and the subsequent results for element $\mathrm{Cr}, \mathrm{Ni}, \mathrm{Co}, \mathrm{C}$ and $\mathrm{O}$ are shown in $\mathbf{d}-\mathbf{h}$, respectively

morphology of the graphene, the details are shown in Fig. 2c, d. Most graphene still existed in a form of wrinkle structure and dispersed on the surface of both $\gamma$ and $\gamma^{\prime}$ phases. It is noted that the location and distribution of the graphene were not dependent on the local microstructure of the matrix, e.g., the graphene could disperse on the surface 
of either one $\gamma$ grain or several, indicating that the attachment between graphene and the matrix was not exclusive. Further analysis using TEM revealed that at the phase interface, the graphene exhibited a 2D layer structure and such structure became curled during thermal processing. Besides, no impurity or porosity could be found on the attaching interface between graphene and the superalloy matrix.

The distribution of the graphene in a 3D scale inside the superalloy matrix was characterized using X-ray tomography, the details is shown in Fig. 3.

It could be seen from Fig. 3 that the graphene was dispersed rather uniformly inside the superalloy matrix. The reconstructed 3D morphology of the graphene exhibited a wrinkled structure, which agreed well with TEM results.

\subsection{Interfacial Bonding Mechanism}

To disclose the interfacial bonding mechanism between graphene and the superalloy matrix, HRTEM characterization is performed and the results are shown in Fig. 4. The graphene in this sample was found right on the edge and exhibited a curling morphology. The EDS measurement along the line as shown in Fig. 4c clearly indicated a transition from the superalloy matrix to the graphene, i.e., shifting of elements from $\mathrm{Ni}, \mathrm{Cr}$ and $\mathrm{Co}$ to $\mathrm{C}$ and $\mathrm{O}$ as the distance was away from the matrix. Besides, according to Fig. 4g, the increase of the $\mathrm{C}$ element was not abrupt, or in other words, the $\mathrm{C}$ element increased in a rather continuous manner, indicating a possible presence of a diffused interface.

Figure 5 shows the diffusion of $\mathrm{C}$ atoms inside the superalloy matrix. As shown in Fig. 5b, the EDS spectrum clearly indicated the existence of the graphene phase at the location as marked by "P" (Fig. 5a). As shown in Fig. 5c or $d$, from the graphene to the superalloy matrix, the microstructure clearly experienced a transition from a hexagonal pattern into a metallic ordering. The color-mask image as shown in Fig. $5 \mathrm{~d}$ also revealed that at the graphene and superalloy interface, the transition of the atoms was in a diffusing manner. A possible bonding mechanism is thus via chemical fusion, but further investigation must be performed to clarify this.

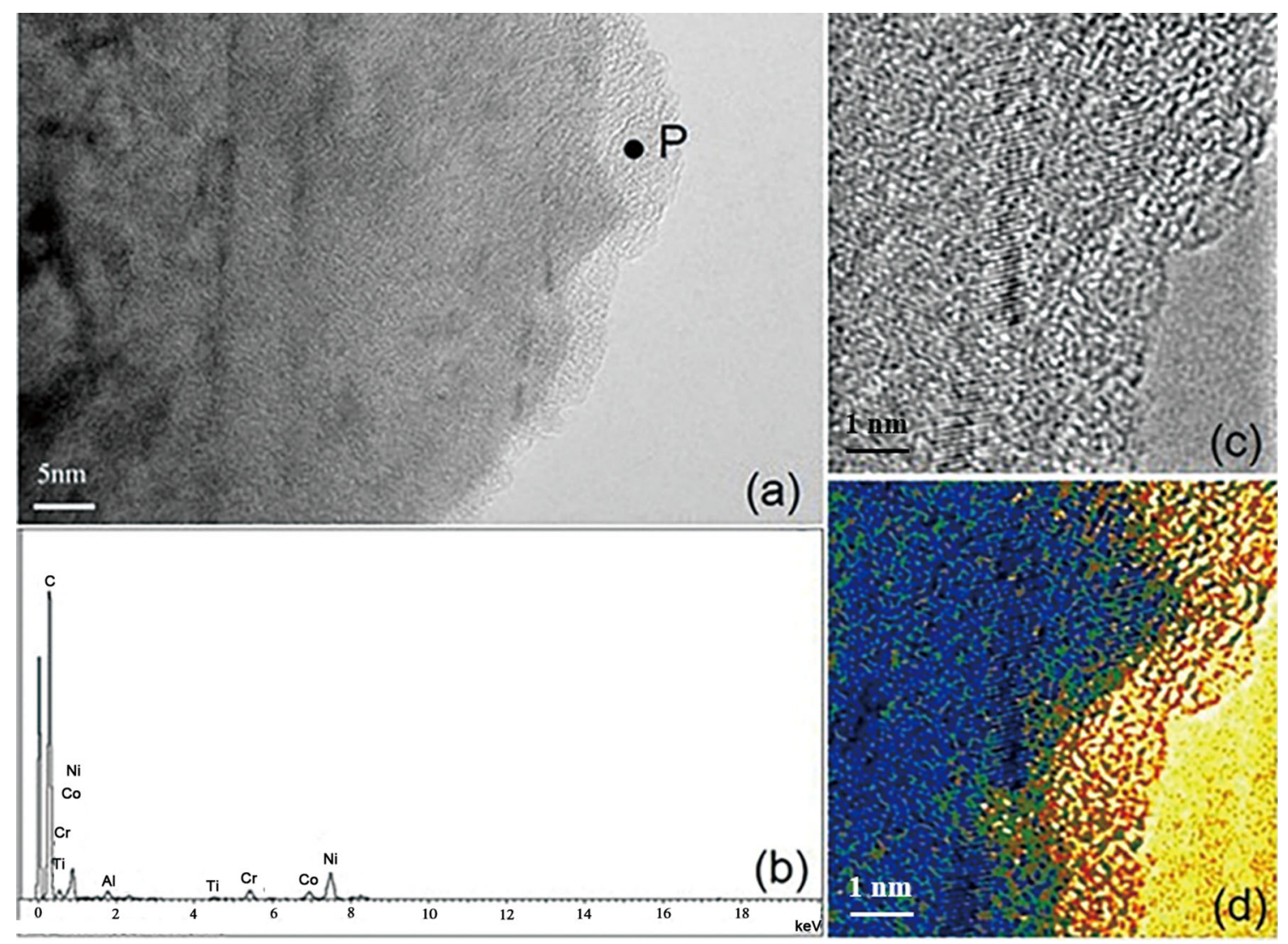

Fig. 5 TEM images showing bonding interface between graphene and superalloy matrix $\mathbf{a}$, EDS analysis of position P $\mathbf{b}$ and zoom-in areas near position $\mathrm{P}$ showing with different contrast colors $\mathbf{c}, \mathbf{d}$ 


\subsection{Improvement of the Mechanical Properties}

Taking the $0.1 \mathrm{wt} \%$ graphene-modified FGH96 superalloy for instance, the tensile properties, including tensile strength, plasticity and yield strength, were all improved significantly comparing with those of the general FGH96 superalloy. At room temperature, the tensile strength and yield strength of the $0.1 \mathrm{wt} \%$ graphene-modified FGH96 are $1626 \mathrm{MPa}$ and $1222 \mathrm{MPa}$, respectively, which are $\sim 3.7$ and $3.6 \%$ higher than those of unmodified FGH96, as shown in Table 2. At high temperature $\left(650{ }^{\circ} \mathrm{C}\right)$, the tensile strength, yield strength and elongation of 0.1 wt\% graphene-modified FGH96 are $1519 \mathrm{MPa}$, $1088 \mathrm{MPa}$ and $26 \%$, respectively, which are $\sim 3.9,2.6$ and $18.7 \%$ higher than those of the unmodified FGH96. More details of which are shown in Table 2, in which each value was an average based on the testing results of three or more samples after heat treatment. The excellent tensile properties of the graphene, together with the uniform dispersity of the graphene inside the matrix were believed to be the major reason for such improvement. The distribution of the graphene on the fracture surface of the sample further verified this postulate, results of which are shown in Fig. 6. Furthermore, the tensile strength decreases with the increase of deformation temperature, this is because high temperature can promote the mobility of the dislocation [30].

Figure 6 shows typical morphologies of fracture surface for a $0.1 \mathrm{wt} \%$ graphene-modified superalloy. On each fracture surface, more graphene are exhibited and acted as a connecting medium or phase so that more pulling force could be endured during the tensile test.

Figure 7 shows the true stress-strain curves of FGH96 superalloys and its graphene-modified composites compressed at different temperatures and strain rates. The significant improvement in hot compression property was observed. It can be seen that the maximum stress was found to decrease as the strain rate increased for both graphenemodified and unmodified FGH96 shown in Fig. 7a. Moreover, at same deformation temperature and the same

Table 2 Tensile properties of FGH96 superalloy and graphene (GR)/FGH96 composites ( $\Psi$ : shrinkage on cross section)

\begin{tabular}{llllll}
\hline Material & Temperature $\left({ }^{\circ} \mathrm{C}\right)$ & Tensile strength $(\mathrm{MPa})$ & Elongation $(\%)$ & $\Psi(\%)$ & Yield strength $(\mathrm{MPa})$ \\
\hline $0.1 \mathrm{wt} \%$ GR/FGH96 & 20 & 1626 & 22.0 & 37 & 1222 \\
FGH96 & 20 & 1568 & - & 21 & 1179 \\
$0.1 \mathrm{wt} \%$ GR/FGH96 & 650 & 1519 & 26.0 & 27 & 1088 \\
FGH96 & 650 & 1461 & 21.9 & 18.5 & 1060 \\
\hline
\end{tabular}
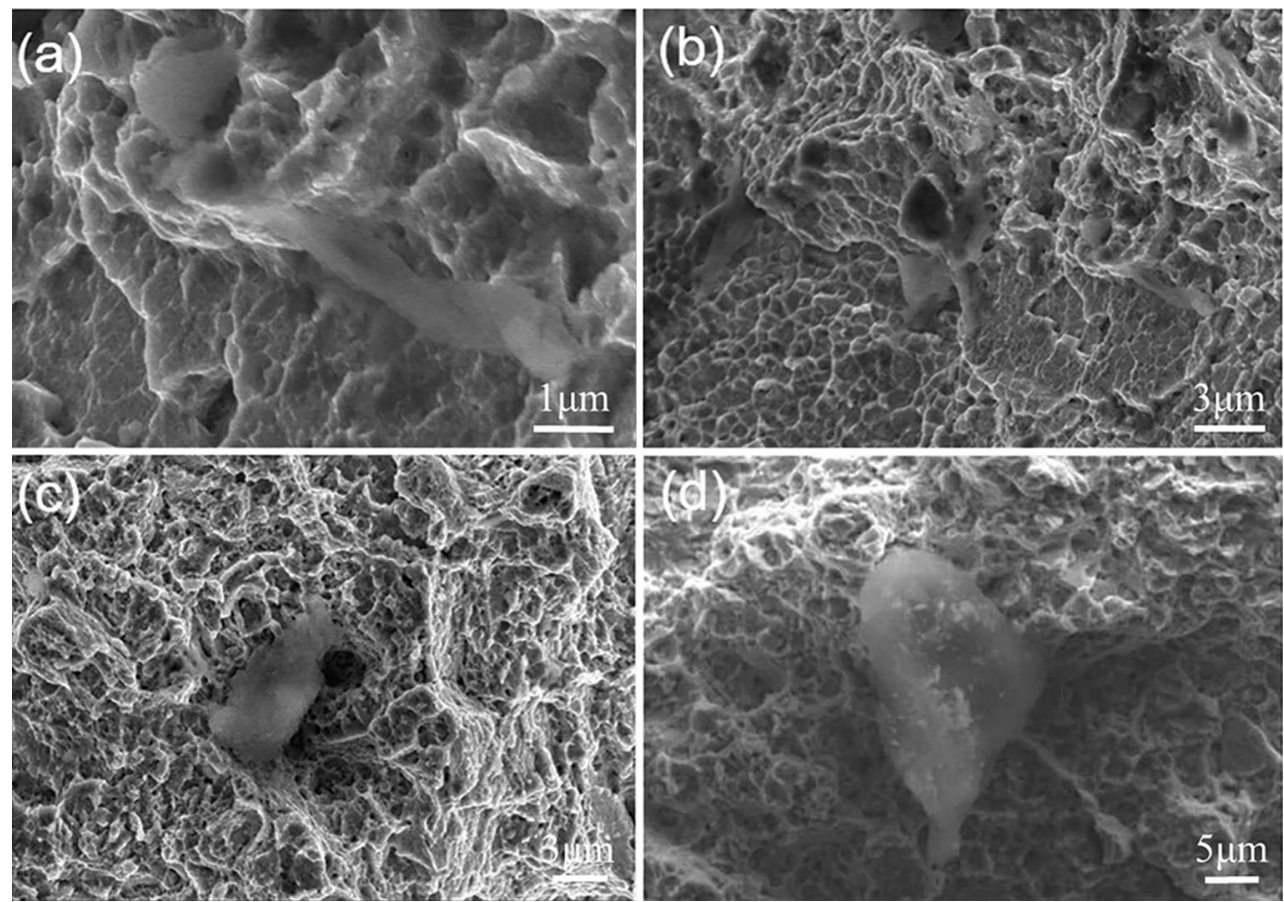

Fig. 6 SEM images of tensile fracture for $0.1 \mathrm{wt} \%$ graphene-modified FGH96 superalloy: a, b fracture morphologies without graphene; c, d fracture morphologies including graphene 

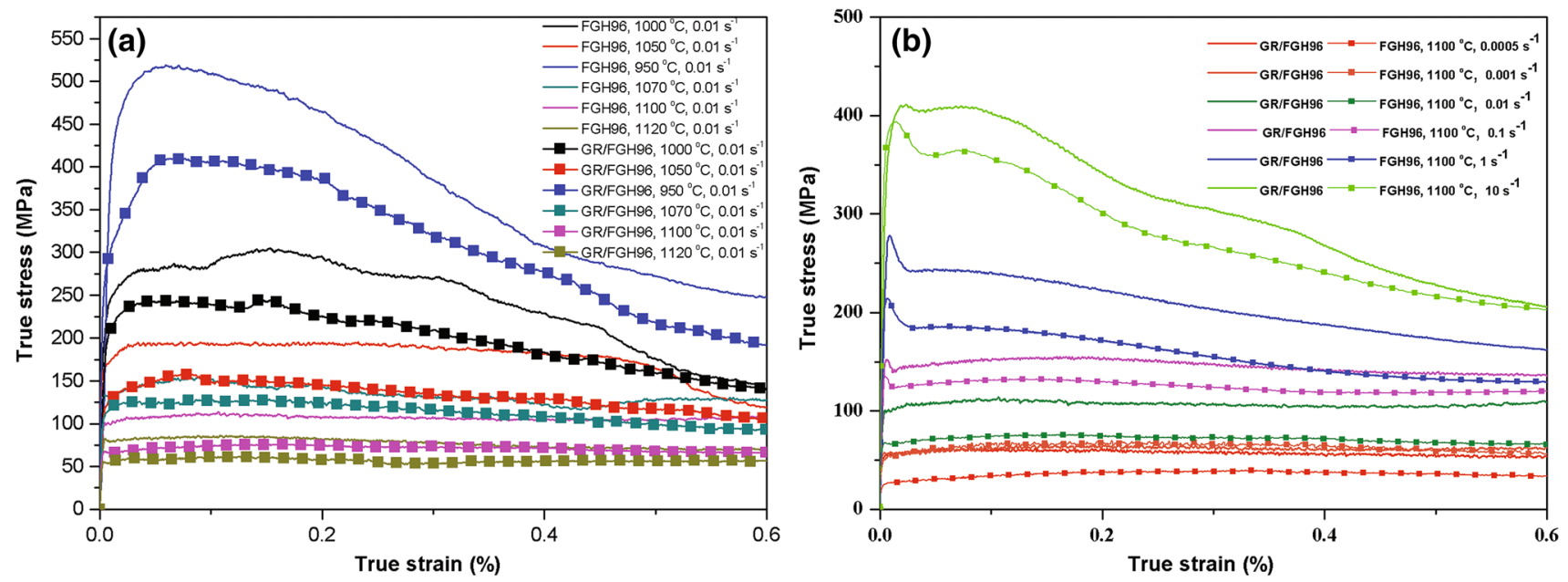

Fig. 7 Hot compression properties of FGH96 superalloy and GR/FGH96 composite under different temperatures a, strain rates b

Table 3 Fatigue properties of FGH96 superalloy and GR/FGH96 composite

\begin{tabular}{|c|c|c|c|c|c|c|}
\hline Material & Temperature $\left({ }^{\circ} \mathrm{C}\right)$ & Waveform & $F(\mathrm{~Hz})$ & $R_{\varepsilon}=\varepsilon_{\min } / \varepsilon_{\max }$ & $\Delta \varepsilon_{\mathrm{t}}=\varepsilon_{\max }-\varepsilon_{\min }$ & $N(\mathrm{cyc})$ \\
\hline $0.1 \mathrm{wt} \%$ GR/FGH96 & 650 & Triangular wave & 0.33 & 0.05 & 0.8 & 126,194 \\
\hline FGH96 & 650 & Triangular wave & 0.33 & 0.05 & 0.8 & $\geq 5000$ \\
\hline
\end{tabular}

$F$ : frequency; $\varepsilon_{\min }:$ minimum strain in one cycle; $\varepsilon_{\max }:$ maximum strain in one cycle; $N$ : fatigue life

strain rate, the magnitude of the peak flow stress of the graphene-modified alloy is higher than the unmodified one shown in Fig. 7a. In Fig. 7b, the compression temperature was kept at $1100{ }^{\circ} \mathrm{C}$ while strain rate varies from $0.0005 \mathrm{~s}^{-1}$ to $10 \mathrm{~s}^{-1}$, flow stress of the graphene-modified FGH96 is significantly higher than unmodified one.

With $0.1 \mathrm{wt} \%$ graphene addition, the GR/FGH96 composite improves its fatigue properties significantly, reaching 126,194 cyc, which is 25 times of the unmodified FGH96, the details are shown in Table 3.

It can be seen in Fig. 8 that $0.1 \mathrm{wt} \%$ addition of graphene (FGH96-0.1 wt\% GR) results in lower residual strain after $125 \mathrm{~h}$; however, the divergency of the residual strain is not obvious before $125 \mathrm{~h}$, which indicates that compared with traditional FGH96 alloy, graphene-modified FGH96 alloy shows a better high-temperature creep properties.

The significant improvement of mechanical properties of graphene-modified FGH96 can be attributed to following four reasons. Firstly, the graphene has extremely high mechanical properties, e.g., the tensile strength is $\sim 130$ $\mathrm{GPa}$ and elastic modulus is $\sim 1.02 \mathrm{TPa}[4,6]$, which are essential for the improvement of the mechanical properties of the superalloy. Secondly, numerous dislocations are produced in the composite due to significant difference of thermal expansion and elastic modules between the graphene and the FGH96 matrix [5, 31]. Thirdly, the load transfer strengthening is another reinforcement factor

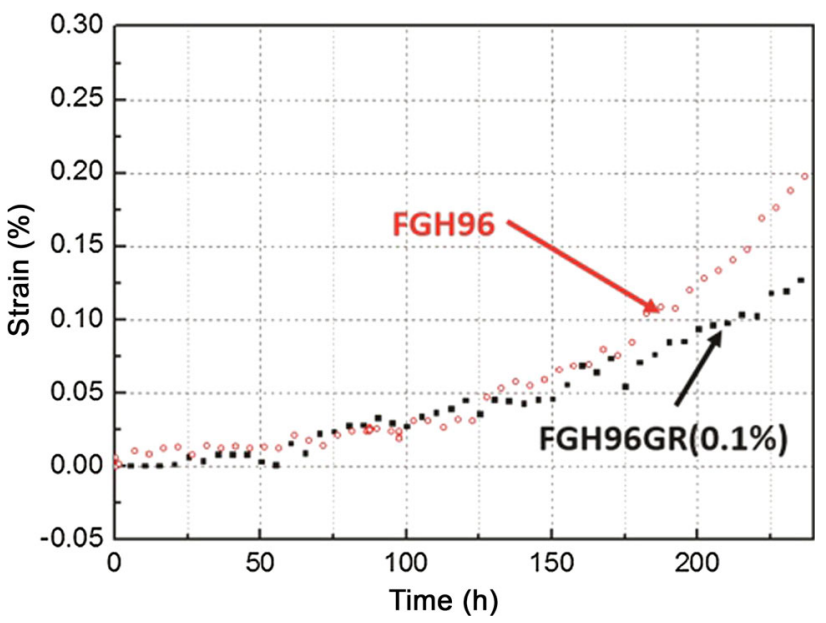

Fig. 8 Creep properties of FGH96 superalloy and GR/FGH96 composite

which can be explained by the shear-lag model [32]. Load transfer from the matrix to graphene is strongly dependent on the interfacial bonding between the matrix and graphene, which is chemical fusion instead of a mechanical one for the graphene and the matrix, as shown in Fig. 4. In addition, most chemical elements in the superalloy cannot move through the graphene sheets [2, 33], which makes graphene as a diffusion barrier in the matrix. This in turn 
effectively resists the degeneration of the mechanical properties of the GR/FGH96 composite.

\section{Conclusions}

A systematical characterization of the bonding mechanism between the graphene and the PM superalloy matrix was performed using OM, SEM, TEM and X-ray tomography. The following conclusions can be drawn:

(1) The graphene-modified FGH96 superalloy can be successfully prepared by employing the so-called wet mixing method. The microstructure observation revealed that the graphene could be dispersed uniformly inside the matrix of the superalloy.

(2) The bonding interface between graphene and the superalloy was shown to be a diffusion instead of abrupt distinction, suggesting that the interface was formed, more possibly, via chemical fusion rather than a mechanical combination.

(3) The uniform dispersion of the graphene inside the matrix could improve the tensile properties of the superalloy significantly. The existence of the graphene on the fracture surface further verified that the graphene acted as a connecting medium so that the effective ability of the bearing load of the material could be improved.

\section{References}

[1] K.S. Novoselov, A.K. Geim, S.V. Morozov, D. Jiang, Y. Zhang, S.V. Dubonos, I.V. Grigorieva, A.A. Firsov, Science 306, 666 (2004)

[2] A.K. Geim, K.S. Novoselov, Nat. Mater. 6, 183 (2007)

[3] Y.P. Bliokh, V. Freilikher, S. Savel'Ev, F. Nori, Phys. Rev. B 79, 7715 (2012)

[4] X. Shen, Z. Wang, Y. Wu, X. Liu, Y.B. He, J.K. Kim, Nano Lett. 16, 3585 (2016)

[5] C. Lee, X. Wei, J.W. Kysar, J. Hone, Science 321, 385 (2008)
[6] J. Chen, C. Jang, S. Xiao, M. Ishigami, M. Fuhrer, Nat. Nanotechnol. 3, 206 (2007)

[7] A.A. Balandin, S. Ghosh, W. Bao, I. Calizo, D. Teweldebrhan, F. Miao, C.N. Lau, Nano Lett. 8, 902 (2008)

[8] M. Liu, C. Chen, J. Hu, X. Wu, X. Wang, J. Phys. Chem. C 115, $25234(2011)$

[9] K. Wang, Y. Wang, Z. Fan, J. Yan, T. Wei, Mater. Res. Bull. 46, $315(2011)$

[10] T. Kuilla, S. Bhadra, D. Yao, N.H. Kim, S. Bose, J.H. Lee, Prog. Polym. Sci. 5, 1350 (2010)

[11] A. Yasmin, J.J. Luo, I.M. Daniel, Compos. Sci. Technol. 66, 1182 (2006)

[12] H. Porwal, P. Tatarko, S. Grasso, J. Khaliq, I. Dlouhý, M.J. Reece, Carbon 64, 359 (2013)

[13] H. Kim, A.A. Abdala, C.W. Macosko, Macromolecules 43, 6515 (2010)

[14] X. Huang, Chem. Soc. Rev. 41, 666 (2012)

[15] J. Du, B. Wen, Appl. Mater. Today 7, 13 (2017)

[16] M. Rashad, F. Pan, M. Asif, L. Li, Prog. Nat. Sci. Mater. 25, 276 (2015)

[17] A. Esawi, K. Morsi, Compos. A 38, 646 (2007)

[18] M. Rashad, F. Pan, Y. Liu, X. Chen, H. Lin, R. Pan, M. Asif, J. She, J. Magnes, J. Magnes. Alloys 4, 270 (2016)

[19] M. Rashad, F. Pan, M. Asif, X. Chen, J. Magnes. Alloys 5, 271 (2017)

[20] M. Rashad, F. Pan, M. Asif, Mater. Sci. Eng. A 644, 129 (2015)

[21] M. Rashad, F. Pan, J. Zhang, M. Asif, J. Alloys Compd. 646, 223 (2015)

[22] F. Chen, J. Ying, Y. Wang, S. Du, Z. Liu, Q. Huang, Carbon 96, 836 (2016)

[23] X.N. Mu, H.M. Zhang, H.N. Cai, Q.B. Fan, Z.H. Zhang, Y. Wu, Z.J. Fu, D.H. Yu, Mater. Sci. Eng. A 687, 164 (2017)

[24] M. Rashad, F. Pan, A. Tang, Y. Lu, M. Asif, S. Hussain, J. She, J. Gou, J. Mao, J. Magnes Alloys 1, 242 (2013)

[25] M. Rashad, F. Pan, D. Lin, M. Asif, Mater. Des. 89, 1242 (2016)

[26] S. Yan, C. Yang, Q. Hong, J. Chen, D. Liu, S. Dai, J. Mater. Eng. 4, 1 (2014)

[27] L.Y. Chen, H. Konishi, A. Fehrenbacher, C. Ma, J.Q. Xu, H. Choi, H.F. Xu, F.E. Pfefferkorn, X.C. Li, Scr. Mater. 67, 29 (2012)

[28] J. Wang, Z. Li, G. Fan, H. Pan, Z. Chen, D. Zhang, Scr. Mater. 66, 594 (2012)

[29] M. Yang, S.M. Xiong, Z. Guo, Acta Mater. 92, 8 (2015)

[30] S. Tian, H. Zhou, J. Zhang, H. Yang, Y. Xu, Z. Hu, Mater. Sci. Eng. A 279, 160 (2000)

[31] E.M. Francis, B.M.B. Grant, J.Q.D. Fonseca, P.J. Phillips, M.J. Mills, M.R. Daymond, M. Preuss, Acta Mater. 74, 18 (2014)

[32] A. Kelly, W.R. Tyson, J. Mech. Phys. Solids 13, 329 (1965)

[33] J.S. Bunch, S.S. Verbridge, J.S. Alden, V.D.Z. Am, J.M. Parpia, H.G. Craighead, P.L. Mceuen, Nano Lett. 8, 2458 (2008) 